\title{
CAST3M modelling of dynamic experiments on PWR high burn-up fuel rods equivalent fuel rod modelling approach validation
}

\author{
Caroline Guerin ${ }^{1, *}$, Chantal Cappelaere ${ }^{2}$, and Marc Ton-That ${ }^{3}$ \\ ${ }^{1}$ Den-Service d'études mécaniques et thermiques (SEMT), CEA, Université Paris-Saclay, 91191 Gif-sur-Yvette, France \\ 2 CEA, DEN, DMN F-91191 Gif-sur-Yvette, France \\ ${ }^{3}$ EDF, DT F-69363 Lyon 07, France
}

Received: 11 October 2019 / Accepted: 15 June 2020

\begin{abstract}
Spent fuel transport and handling operating rules exclude any accidental risk. However, in the framework of the PRECCI R\&D project, the impact on a spent fuel rod is taken into account. This study was carried out in CEA laboratories with the funding and the technical support of EDF. Since fuel rods are slender structures, it is of particular interest to model them with one dimensional finite elements. While the zirconium alloy elasto-plastic law can be directly used for the plugs and plenum regions, an equivalent stress-strain law is necessary for the fuel column level, especially in the case of high burnups since the contact between the pellets and the clad reinforce the contribution of the fuel to the rod mechanical behavior. The equivalent law as well as the correlation between the clad and the equivalent plastic strain were established from CAST3M finite element modelling of four point bending of fuel rods segments. This model, meshed with three dimensional elements, takes into account elasto-plastic behavior for the clad and cracking of the $\mathrm{UO}_{2}$ fuel pellets. Bending experiments on spent fuel rods performed at LCMI laboratory allowed to validate this modelling as reported in a previous article. The axial dynamic load impact tests performed by Hirose et al. on PWR fuel rods specimens were modelled with CAST3M with one dimensional elements using the determined equivalent law. The maximum loads calculated with the equivalent law are in excellent agreement with the experimental results. The deformed shapes as well as the failure modes are also in good agreement. The maximum calculated strains reach the fracture strain of the rods at the location where the failures are observed experimentally. This study reinforces the confidence in the equivalent law. It moreover indicates that zircaloy behavior for dynamic loadings can be modelled with the laws established at lower strain rates, as suggested by the moderate variation of the mechanical properties as a function of strain rates determined by dynamic tensile tests from literature.
\end{abstract}

Keywords: Spent fuel rod / dynamic loading / rod integrity / CAST3M

\section{Introduction}

The spent fuel transport and remote handling follow processes excluding any accident. However, in the framework of the PRECCI R\&D project, we developed an approach based on experiments and calculations that allowed us to perform finite element calculations using one dimensional elements with a behavior equivalent to that of the fuel rod and to estimate the cladding integrity. Since fuel rods are slender structures, it is indeed of particular interest to model them with beams in finite elements

\footnotetext{
* e-mail: caroline.guerin@cea.fr
}

calculations. All modellings were performed with the finite element solver CAST3M [1]. A fuel rod segment model with the clad and the pellets meshed with three-dimensional elements and taking into account the elasto-plastic behavior for the clad and the cracking of the $\mathrm{UO}_{2}$ fuel pellets was first developed. Three points bending experiments on spent fuel rods performed at LCMI laboratory allowed previously to validate this modelling [2]. Since the circular curvature obtained in four points bending test allows to determine easily the equivalent strain than in the case of three points bending test, modelling of such experiment was then performed using the before mentioned finite element model of the rod segment. The equivalent stress-strain law as well as the fracture strains of the fuel 


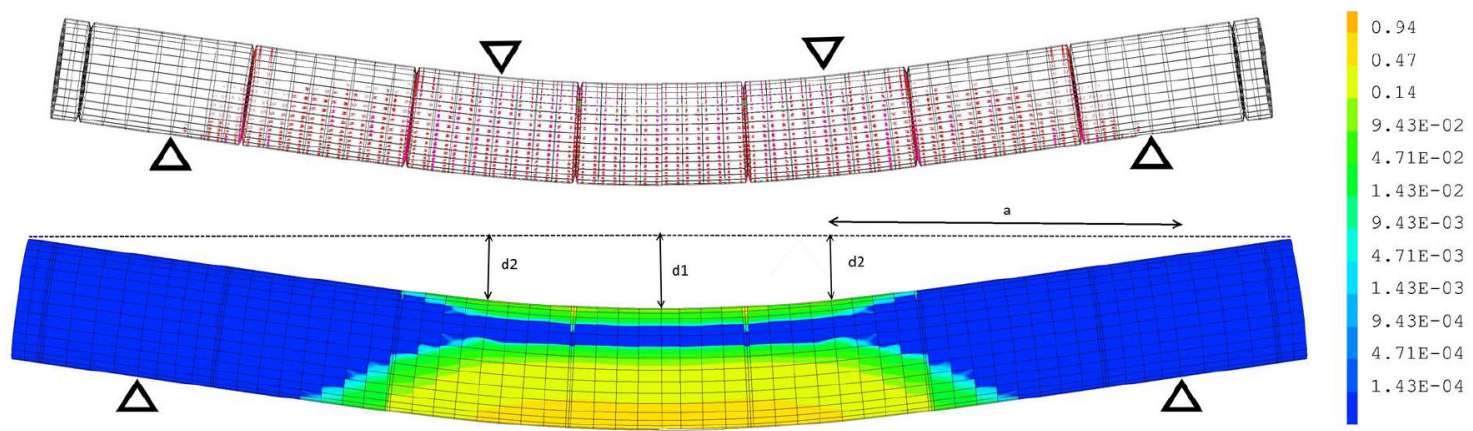

Fig. 1. Cracks openings (top) in the pellets displayed in red and inelastic strain (bottom) in the clad (normalized to the fracture strain of the clad) resulting from four points bending of a rod segment. The support pins and loading pins are displayed as triangles.

rods at the column level were obtained from calculations of four point bending of the fuel rod segment. The resulting equivalent law was then used to model fuel rod segments with one dimensional elements. The experiments performed at the NRA by Hirose et al. [3,4] and consisting in axial dynamic loads impact on high burnup PWR fuel rod segments were calculated with this second model. The maximal loads as well as the fracture modes obtained from modelling and experiments were compared. The maximum equivalent inelastic strain calculated was compared with the fracture strain in the rod in order to study the fuel rods integrity. This is of particular interest since cladding constitutes the first confinement barrier.

\section{Four points bending of a spent fuel rod, equivalent behavior determination}

CAST3M modelling of the fuel rod bending was performed with a three dimensional mesh of the clad and the pellets. The elasto-plastic behavior established from static tensile test was taken into account for the irradiated clad. Fracture strains for the irradiated clads were also obtained from these tests. For the pellets, the Ottosen fictitious crack model [5] suitable for brittle material was used with parameters corresponding to the $\mathrm{UO}_{2}$ characteristics. Unilateral support was considered between successive pellets. Different boundary conditions can be considered between the pellets and the clad. Firstly, tight contact due to high burn up can be modelled by merging the maximum diameters points of the pellets with the cladding inner mesh. Secondly, gap between the clad and pellet can be modelled with (unilateral) support between the nodes of the two meshes. In the case of the gap, the pellets at the extremities of the rod segment have external nods merged to the clad in order to sustain axially the column. As previously reported in [2], this modelling was validated by comparison of the calculated results with those obtained from three points bending experiments performed on a sixcycle fuel rod segments at the LECI facility in Saclay: laboratory for studies on irradiated materials. Four points bending of a rod segment was then calculated using this three dimensional modelling of fuel rod segment in order to determine its equivalent behavior. For the maximum imposed displacement at the loading pins symbolized with triangles pointing downwards, the upper section of Figure 1 shows the shape of the pellets with the cracks openings while the lower section shows the shape of the clad with the normalized inelastic strain. Support pins are symbolized with triangles pointing upwards.

The equivalent stress-strain law is obtained from $F\left(d_{1}\right.$, $d_{2}$ ), where $F$ is the calculated reaction strength at the loading pins $\left(F / 2\right.$ at each loading pin), $d_{1}$, the displacements at the midpoint and $d_{2}$, the displacement at the loading pins (as displayed in Fig. 1).

The strength-displacement function is converted in stress strain curve, $\sigma(\varepsilon)$, considering the circular shape of the rod between the two loading pins (radius of curvature $R$ ). The strain is expressed from the rod radius, $r$, and $R$ from :

$$
\varepsilon=\frac{r}{R}=\frac{r}{\frac{d_{1}-d_{2}}{2}+L^{2}\left(d_{1}-d_{2}\right)},
$$

where $L$ is the distance between the two loading pins.

The stress in outer fibres at midpoint is calculated from the momentum $M$ and the moment if inertia $I_{z}$ :

$$
\varepsilon=\frac{M r}{I_{z}}=\frac{2 F a}{\pi r^{3}}
$$

where $a$ is the distance between the loading pin and the nearest support pin as shown in Figure 1.

The equivalent stress-strain laws, $\sigma(\varepsilon)$, obtained in the case of perfect contact and in the case of a gap between clad and pellets are displayed in Figure 2 for two different clad alloys. We chose to normalise the stress and strains with the fracture stresses and strains of the clad divided by 2 and not with the maximum stresses and strains of the rod segments in order to visualise the higher rigidity of the systems with merged points compared to that with the gap between the pellets and the clad. In the case of perfect contact, one may observe at low strain a decrease in the slope due to the fuel cracking initiation. When there exists a gap between the clad and the pellet, the stiffness of the rod is lower and this decrease is not observed.

The inelastic strain in the clad is plotted as a function of the inelastic equivalent strain in Figure 3. For the lower equivalent strain, the strain in the clad remains equal to 


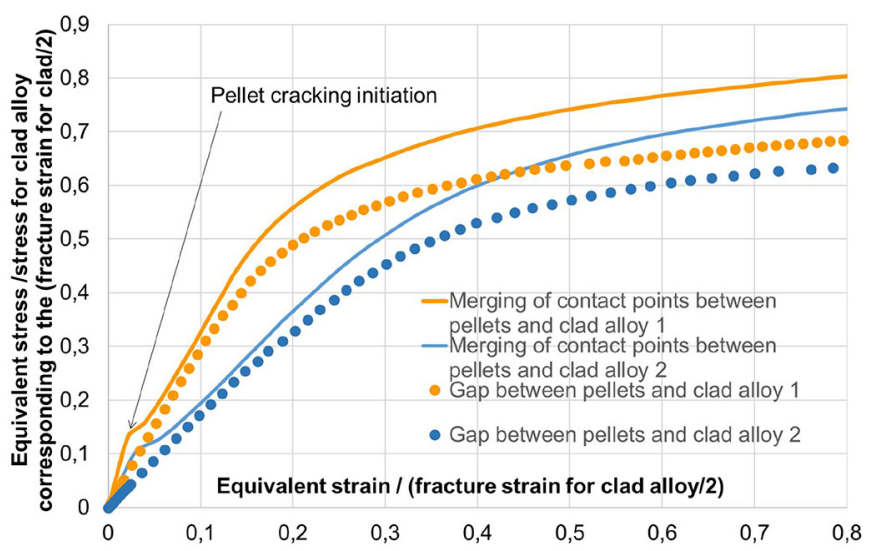

Fig. 2. Equivalent stress-strain law obtained from four points bending calculations of rod segments with different clad alloys and clad-pellets boundary conditions (strains normalized to half of the fracture strains of the clad alloys and strength normalised to strength values at these strains).

zero since the inelastic equivalent strain is due only to pellets cracking. A change in the slope is observed in the case of the gap due to the different stages of gap closure during bending.

The bending calculations were performed up to the maximum loading for which convergence of calculation can be reached. For the maximum loading, the cracking of the pellets is important as well as the plastic strain in the clad which reaches $75-97 \%$ of the fracture strain of the clad. For a more important bending above this loading, the pellets cannot hold the load any longer and the load is transferred to the clad that cannot hold it neither, leading to non convergence. We therefore consider that the fuel rod integrity is insured up to the maximum equivalent strain determined from the four points bending modelling. Let us for instance consider the case of the merging of contact points between clad and pellets. As indicated by the arrows in Figure 3, the inelastic equivalent fracture strain of the fuel rod is equal to 0.5 of the fracture strain of the clad for the rod with the clad alloy 1 and it is equal to 0.67 of the fracture strain of the clad for the rod with the clad of alloy 2.

For their subsequent use, the stress-strain equivalent laws were linearly extrapolated at higher strain values.

\section{Dynamic experiment calculations}

For the assessment of high burnup spent fuel integrity at dry cask drop accident, dynamic behavior of PWR fuel rods was investigated by Hirose et al. [3,4]. Partial fuel rod specimens with Zy-4/MDA (Mitsubishi developed Alloy) (Zr-0.8Sn-0.2Fe-0.1Cr-0.5Nb) claddings (stress relieved) with burnup $(52-55 \mathrm{GWj} / \mathrm{tU})$ were tested with axial dynamic load in order to obtain the threshold of fuel rod failure. As reported in Table 1, four fuel section specimens and one plenum upper specimen (including pellets) were tested using $3 \mathrm{~kg}$ weight and impact speed in the range of 8 $11 \mathrm{~m} / \mathrm{s} .2$ short length specimens (total length: $298 \mathrm{~mm}$ including two plugs each of $35 \mathrm{~mm}$ long) and 3 long length

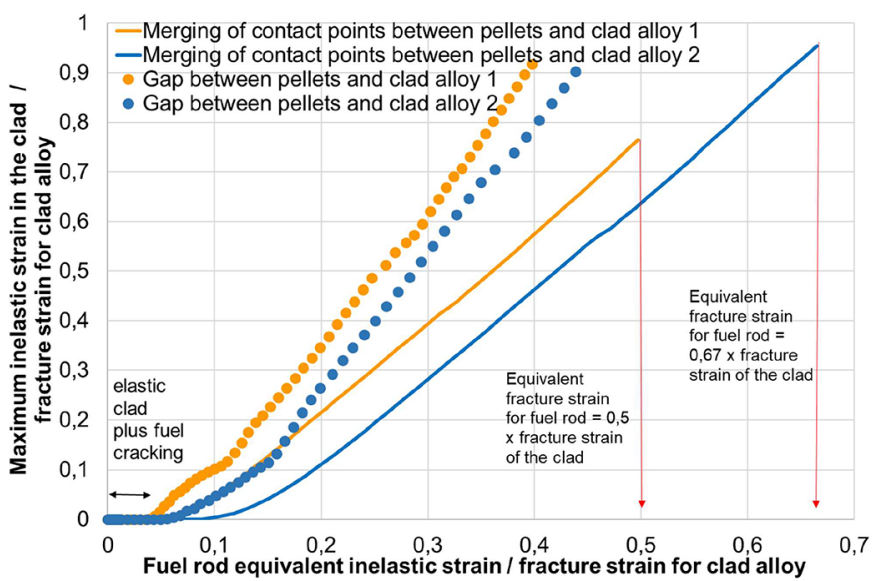

Fig. 3. Maximum inelastic strain in the clad as a function of the equivalent inelastic strain calculated from four points bending of rods with different clad alloys and clad-pellets boundary conditions. Strains are normalized to the fracture strains of the clad.

(total length: $523 \mathrm{~mm}$ including two plugs each of $35 \mathrm{~mm}$ long plus a plenum in the case of sample long 1) were tested to study the influence of the rod length on dynamic behavior. These experiments were modelled with CAST3M. The rods segments were meshed using unidimensional elements. The plugs and the plenum $(16 \mathrm{~cm}$ long) were respectively modelled with beam and pipe elements following the elasto-plastic behavior of the clad alloy while the determined equivalent law was used for the length of the rod corresponding to the fuel column. Calculations were performed for the two clad alloys but only in the case of perfect contact between clad and pellets since experiments were only performed on high burnup rods specimens. The axial direction of the rod is set along the $y$ direction. Displacements of the rod segment are restrained to the $(O, x, y)$ plane and the lower point of the rod is locked while that of the upper point is set equal to zero along the $x$ direction. The initial velocity of the upper point along the $-y$ direction is imposed equal to that of the mass impact. The loading consists in the proper mass of the rod segment plus a concentrated mass of $3 \mathrm{~kg}$ at the upper point.

The variation of the calculated reaction forces at the lower point of the fuel rods is plotted as a function of time in Figure 4 for the different specimens with clad alloy 1 . The maximum calculated forces obtained are reported and compared to the maximal experimental loads obtained for each sample in Table 1 . One may see that these loads are in particularly good agreement in the case of rods with clad alloy 1. As observed experimentally, the length of the specimen does not affect the maximum load while the plenum presence does. Figure 5 displays the inelastic equivalent strain as a function of time. This quantity keeps on increasing up to $7 \mathrm{~ms}$. Failure was experimentally observed in all cases excepted for sample L2 which has however exactly the same characteristics and loadings as sample L3. From the calculations, all samples are supposed to fail since the inelastic equivalent strains normalised by the clad alloy fracture strain are equal or slightly above the 
Table 1. Specimens description, experimental and calculated results for dynamic load impact test.

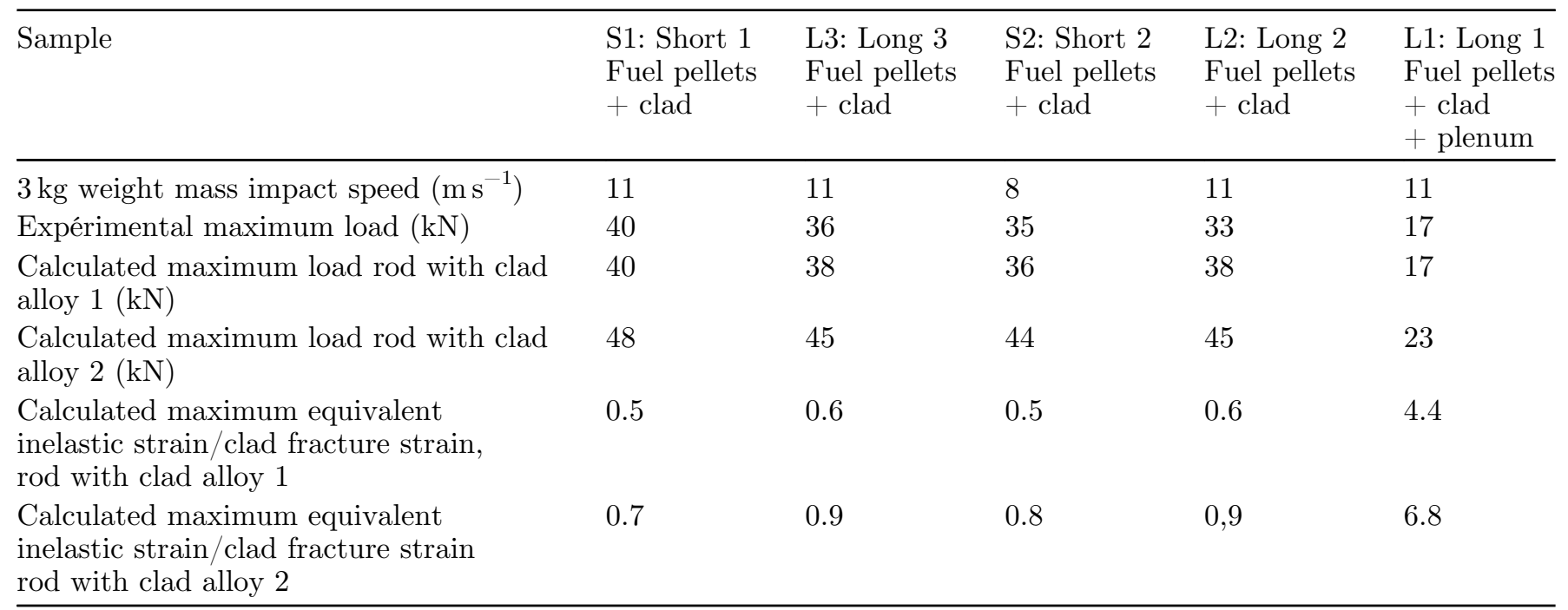

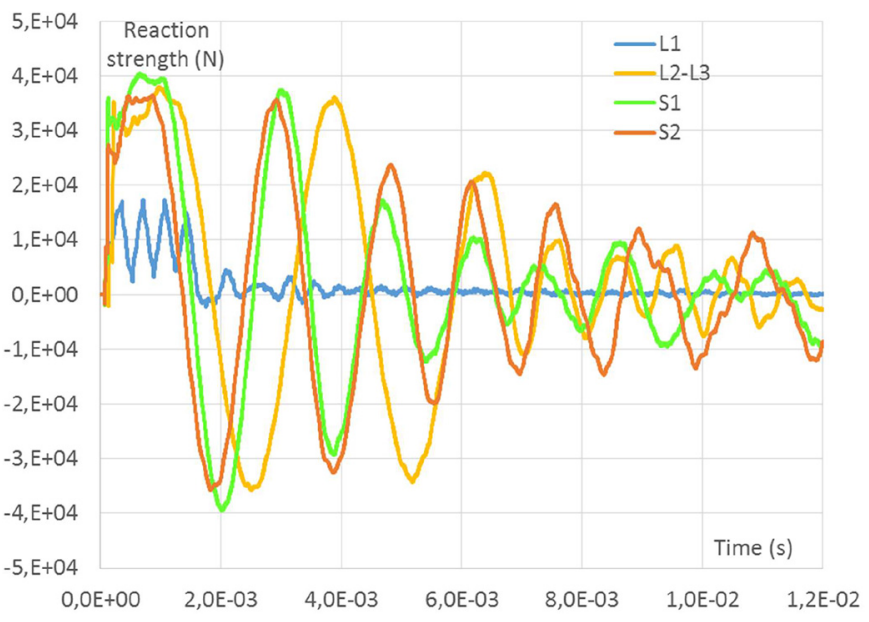

Fig. 4. Variation of the calculated reaction strength at the lower point of the specimen as a function of the time. Case of clad alloy 1 .

limits reported in paragraph 2 ( 0.5 for alloy 1 and 0.67 for alloy 2). In the case of specimen L1, the maximum strain is located in the plenum region. Therefore, the failure should occur in this case when the equivalent strain divided by the fracture strength of the alloy reaches the value of 1 . From Figure 5, rod failure can therefore be predicted around $1.3 \mathrm{~ms}$ after mass impact in the case of L1 and after $6 \mathrm{~ms}$ in the case of the other specimens.

The experimentally observed failure modes and the inelastic strain on the final shapes calculated in the case of specimens with clad alloy 1 are reported in Table 2. The final shapes of the specimens and inelastic equivalent strain distribution obtained in the case of clad alloy 2 are quite similar. Hirose et al. noted that after initial impact, the axial load increased with compression and high-order mode bending occurred, then first-order mode bending occurred. One may see that the final shape calculated seems to

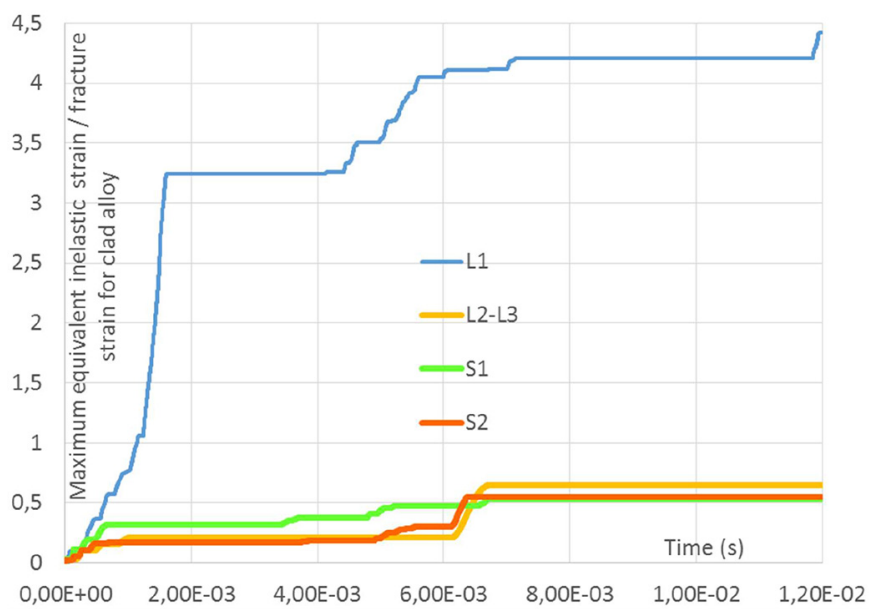

Fig. 5. Variation of the maximum equivalent inelastic strain normalized by the fracture strain of the clad as a function of the time. Case of clad alloy 1.

correspond in each case to the failure mode. A more precise description of the calculated displacements perpendicularly to the axial direction of the specimens can be visualized in Figure 6 for different time $t=1.3 \mathrm{~ms}, t=6 \mathrm{~ms}$ and $t=12 \mathrm{~ms}$ (final time) after impact. The inelastic equivalent strain as a function of elevation at the same times after impact is displayed for the different specimens in Figure 7. From these figures it can be seen that in the case of the S1 and L1 samples the final shape does not correspond to bending mode and is therefore compatible with the experimentally observed shearing failure. For L1 specimen, the maximum strain is calculated in the plenum where failure was experimentally observed. For S1 and S2, the maximum strain is respectively calculated at the midpoint of the specimen and decentered, where failure was observed. For L2 specimen, high order bending failure was experimentally observed and calculated. 
Table 2. Specimens description and experimental and calculated results for load impact test.

\begin{tabular}{llllll}
\hline Specimen name & S1: Short 1 & L3: Long 3 & S2: Short 2 & L2: Long 2 & L1: Long 1 \\
and type & Fuel pellets & Fuel pellets & Fuel pellets & Fuel pellets & Fuel pellets \\
& + clad & + clad & + clad & + clad & + clad + plenum \\
\hline
\end{tabular}

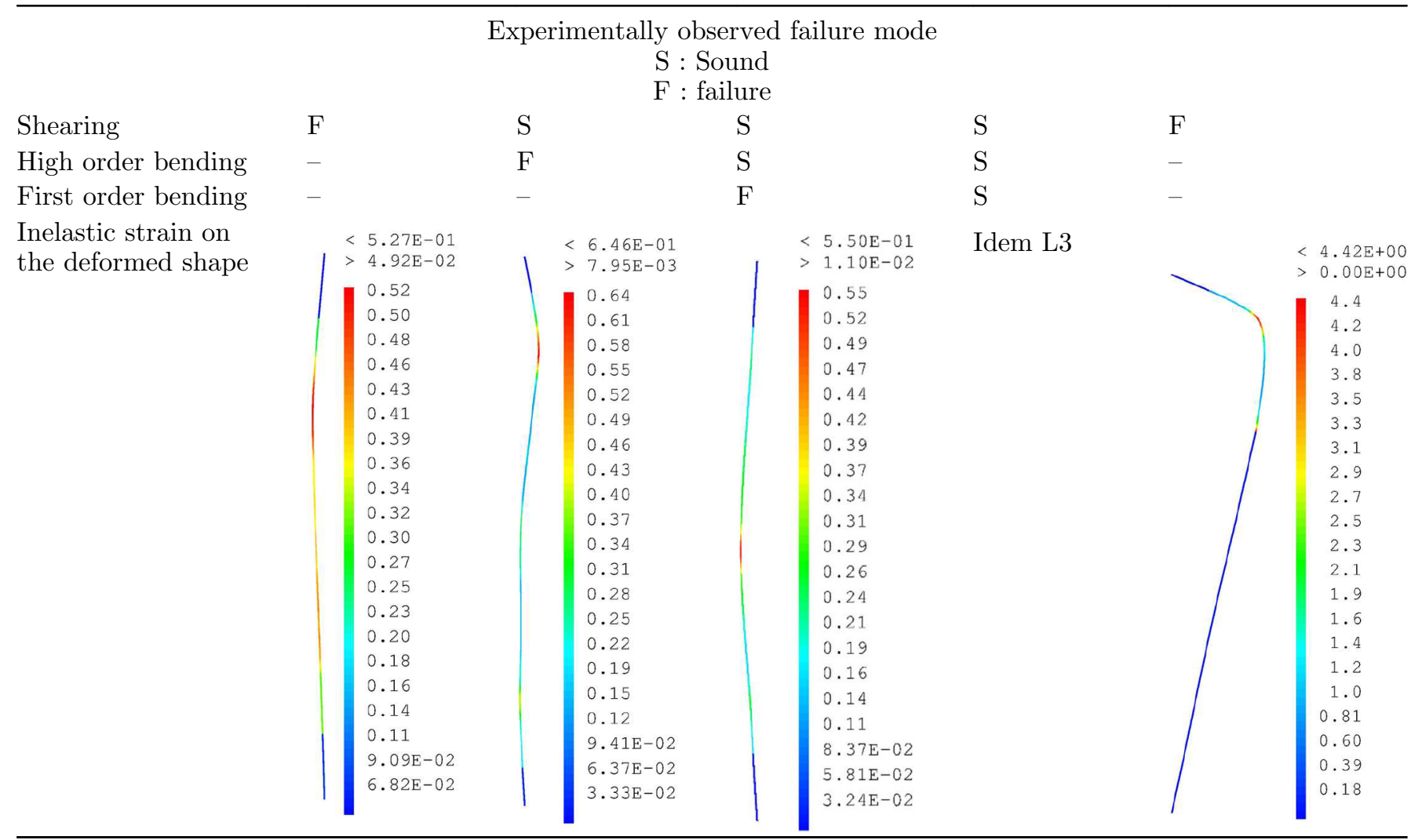

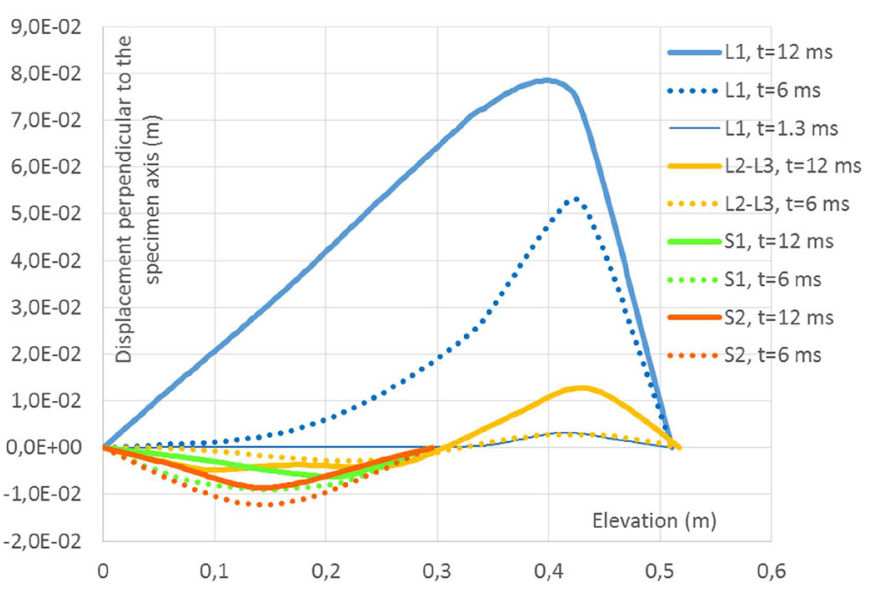

Fig. 6. Displacement perpendicular to the specimen axis as a function of the elevation at time 1.3, 6 and $12 \mathrm{~ms}$. Case of clad alloy 1 .

\section{Conclusions}

Modelling of four points bending of fuel rod segments allowed us to determined equivalent stress-strain laws as

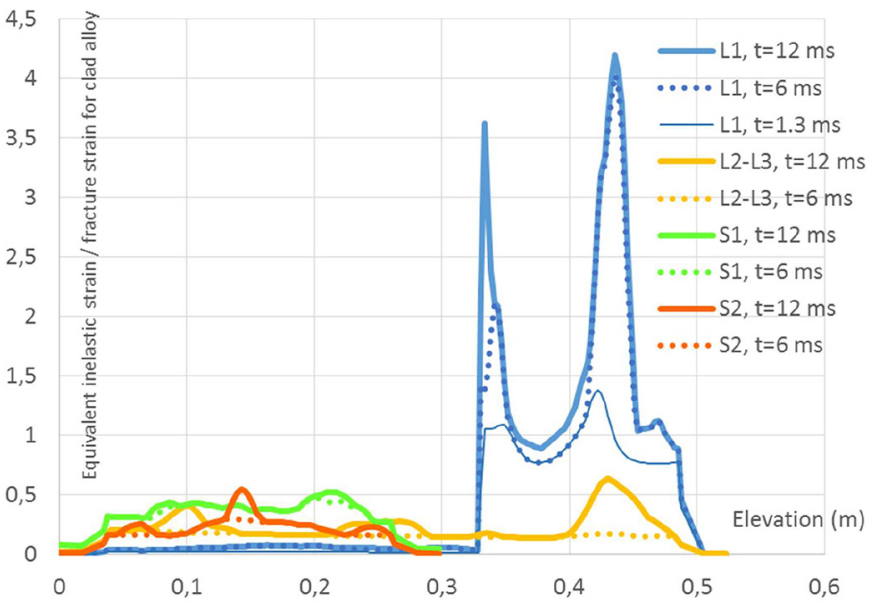

Fig. 7. Equivalent inelastic strain normalized by the fracture strain of the clad as a function of the elevation at time 1.3, 6 and $12 \mathrm{~ms}$. Case of clad alloy 1.

well as failure strain criterions that can be used to model the rods in the fuel column part level with unidimensional finite elements and study their integrity. The constitutive equation was established for different 
clad alloys and in the case of a gap or perfect contact between the clad and the pellets. These last laws, convenient for high burnup, were used to model the experiments of axial dynamic load impact on fuel rods specimens performed at NRA by Hirose et al. The maximum loads calculated are in good agreement with the experimental values. Moreover, the calculated shapes and strains are in good agreement with the experimentally observed failure modes. The equivalent constitutive laws seem therefore convenient to model fuel rods behaviour under dynamic solicitations (calculated strain rates were above $100 \mathrm{~s}^{-1}$ ). As a consequence, it also seems consistent to model the zircaloy behavior for dynamic loadings using the laws established at lower strain rates, as suggested by the moderate variation of the mechanical properties as a function of strain rates determined by dynamic tensile tests and reported in [4]. Future experiments consisting in four points bending of irradiated fuel rods segments will be performed at LECI for strain rates above $10 \mathrm{~s}^{-1}$ and modelled with CAST3M in order to verify these hypotheses. Further development could reside in modelling dynamic behaviour of an irradiated assembly using the equivalent constitutive law for the fuel column level of fuel rods modelled with beam elements.

\section{References}

[1] P. Verpeaux, A. Millard, T. Charras, CASTEM 2000: Une approche moderne du calcul des structures, in J.M. Fouet, P. Ladeveze and R. Ohayon (Eds.), Calcul des structures et Intelligence artificielle, Pluralis, Paris, France (1989) 261-271

[2] C. Guerin, T. Laporte, C. Cappelaere, C. Vaille, P. Mongabure, A. Miquet, P. Bouffioux, CAST3M modelling of a spent fuel assembly bending during a handling accident. Rod failure risk evaluation from the experimental results of spent fuel rod bending test, Mech. Ind. 15, 301-305 (2014)

[3] T. Hirose, M. Ozawa, A. Yamauchi, Fuel rod mechanical behavior under dynamic load condition on high burnup spent fuel of BWR and PWR, in International Conference on Management of Spent Fuel from Nuclear Power Reactors: An Integrated Approach to the Back-End of the Fuel Cycle, 15-19 June 2015, Vienna, Austria. Available at http://www-pub. iaea.org/iaeameetings/cn226p/Session7/ID75HIROSE.pdf

[4] T. Hirose, M. Ozawa, H. Miura, T. Baba, K. Kamimura, Japan Nuclear Energy Safety Organization (JNES), Research on integrity of high burnup spent fuel under long term dry storage and transport. NEA/CSNI/R (2013) 10. Available at https://www.oecd-nea.org/nsd/docs/2013/csni-r2013-10.pdf

[5] O. Dahlblom, N. Ottosen, Smeared crack analysis using generalized fictitious crack model, J. Eng. Mech. 116, 55-76 (1990)

Cite this article as: C. Guerin, C. Cappelaere, M. Ton-That, CAST3M modelling of dynamic experiments on PWR high burn-up fuel rods equivalent fuel rod modelling approach validation, Mechanics \& Industry 20, 808 (2019) 